\title{
Compacton matter waves in binary Bose gases under strong nonlinear management
}

\author{
F.Kh. Abdullaev ${ }^{1}$, M.S.A. Hadi ${ }^{1}$, M. Salerno ${ }^{2}$, and B. Umarov ${ }^{1}$ \\ 1 Deparment of Physics, Kulliyyah of Science, International Islamic \\ University Malaysia, 25200 Kuantan, Pahang, Malaysia and \\ 2 Dipartimento di Fisica "E.R. Caianiello", CNISM and INFN - Gruppo Collegato di Salerno, \\ Universitá di Salerno, Via Giovanni Paolo II, 84084 Fisciano (SA), Italy
}

(Dated: July 31, 2021)

\begin{abstract}
The existence of compacton matter waves in binary mixtures of quasi one-dimensional BoseEinstein condensates in deep optical lattices and in the presence of nonlinearity management, is first demonstrated. For this, we derive an averaged vector discrete nonlinear Schrödinger equation (DNLSE) and show that compacton solutions of different types can exist as stable excitations. Stability properties are studied by linear analysis and by direct numerical integrations of the DNLSE system and their dependence on the inter- and intra-species scattering lengths, investigated. We show that under proper management conditions, compactons can be very robust excitations that can emerge spontaneously from generic initial conditions. A possible experimental setting for compacton observation is also discussed.
\end{abstract}

\section{INTRODUCTION}

It has been recently demonstrated that Bose-Einstein condensates (BEC) in deep optical lattices (OL) when exposed to strong and rapid periodic time modulations of the scattering lengths can support matter wave compactons, i.e. localized excitations with a compact support 1]. Similarly to discrete breathers, compactons are intrinsically localized stable excitations. In contrast with them, however, compactons have no exponential tails, the lacking being due to the effective nonlinear dispersion induced by the modulation, which permits the vanishing of the tunneling just at the compacton edges. In particular, it was shown (see [1] for details) that after averaging out the fast time scale dynamics, the tunneling rate of the resulting averaged system depends not only on modulation parameters but also on local field density differences (atom numbers in the BEC case) between neighboring sites. Thus, the periodic modulation in time of the scattering length (nonlinear management) can be used to change the original dispersion (e.g. the one in absence of management) into an effective nonlinear dispersion essential for the compacton existence.

Field dependent tunneling suppression in the presence of strongly and rapidly modulated interactions was demonstrated in 2] for the case of a BEC trapped in a double well potential (compacton formation being severely restricted in this case by the size of the system) and in 1] for a one-dimensional BEC array modeled by the discrete nonlinear Schrödinger equation (DNLSE). The phenomenon of compacton formation, however, is of general validity and, as we demonstrate in this paper, can occur also in more complicated BEC systems.

From an experimental point of view, periodic time modulations of the scattering length can be achieved by the Feshbach resonance (FR) technique [3], e.g. by varying the external magnetic field near a resonant value. Besides compacton excitations, periodic modulations of the scattering lengths can be used to create density dependent gauge fields [4], this being presently a field of rapidly growing interest, connected with interesting physical phenomena, including pair superfluidity, exactly-defect free Mott-insulator states etc. [5]. Properties of the superfluid -Mott transition in a 2D square and a $3 \mathrm{D}$ cubic optical lattice with periodic modulation of the atomic scattering length have been investigated in [6]. Modulations of the interactions were used also to design new correlatedhopping models for fermions in optical lattices [7] and, in combination with OL shaking, for engineering unconventional Bose-Hubbard models [8].

Spatial and temporal periodic changes of the scattering lengths have been shown to be effective tools to change stability properties of nonlinear excitations, leading to the existence of two dimensional bright solitons in one and two component attractive condensates 9 13]. Moreover, they were used to induce long lived Bloch oscillations 14 16], dynamical localization 17], Rabi oscillations [18] of BEC gap solitons in optical lattices, Faraday waves [19, 20] etc. Nonlinear management techniques were considered also in nonlinear optics to stabilize 2D and $3 \mathrm{D}$ solitons and to reduce collapse in optically layered media with self-focusing interaction [9], to improve communication capacities via soliton dispersion management in optical fibers [21], to create linear superpositions of gap solitons in periodic Kerr media [22], etc.

All these studies refer to the single component case, e.g. BEC made by a a single atomic species. An interesting question to ask, however, is wether compacton excitations could exist also in multi-component systems of interest both for BEC and nonlinear optics. The aim of the present paper is just to provide an answer to this question.

In this respect we introduce an averaged vector DNLSE with effective nonlinear discrete dispersion terms which describes the dynamics of a BEC array in the presence of strong inter-species and intra-species scattering length modulations. The existence of different compacton states in the form of bright-bright (B-B), bright-dark (B-D), and dark-dark (D-D) pairs, is first demonstrated and stability properties of these states investigated both by 
means of a linear stability analysis and by direct numerical integrations of the model equations. As a result we find that while single site and two sites out of phase B-B compactons are stable in the whole parameter range, for the other modes there exists thresholds in the tunneling constant rate below which they cannot exist as stable excitations. The dependence of the compacton stability on inter-species interaction is also investigated. For D-D compactons we find that when the inter-species scattering length is detuned to zero (e.g. in the uncoupled single component limit) the stability becomes narrower compared to that of the pure two components case. The predictions of our analysis are shown to be in good agreement with the results obtained direct numerical simulations of the model. The emergence of compact excitations from generic initial excitations and possible experimental settings for their observation are also discussed.

The paper is organized as follows. In Sec. II we introduce the model equations and discuss the averaged equations. In Section III we derive the conditions for existence of two component of B-B, B-D and D-D compactons and discuss stability properties. In section IV we consider the case of D-D compactons in the uncoupled limit corresponding to the single component dark compacton case. In section $\mathrm{V}$ the emergence of compact excitations from generic initial excitations is investigated and possible experimental parameter design provided. Finally, in Sec. VI the results of the paper are briefly summarized.

\section{MODEL EQUATIONS AND AVERAGING}

Two-component atomic BEC in a deep OL can be described in the tight binding approximation by the following vector DNLSE [23]

$$
\begin{gathered}
i \dot{u}_{n}=-\kappa_{1}\left(u_{n+1}+u_{n-1}\right)-\left(\gamma_{1}\left|u_{n}\right|^{2}+\gamma_{12}\left|v_{n}\right|^{2}\right) u_{n} \\
i \dot{v}_{n}=-\kappa_{2}\left(v_{n+1}+v_{n-1}\right)-\left(\gamma_{12}\left|u_{n}\right|^{2}+\gamma_{2}\left|v_{n}\right|^{2}\right) v_{n}
\end{gathered}
$$

where the overdot stands for time derivative, the coefficients $\kappa_{i}, i=1,2$, are related to the tunneling rates of atoms between neighboring wells of the optical lattice and $\gamma_{12}, \gamma_{i}, i=1,2$ are nonlinear coefficients related to the inter-species $\left(a_{12}\right)$ and intra-species $\left(a_{i i}, i=1,2\right)$ scattering lengths, respectively. Eq. (10) arises also in nonlinear optics where they model the propagation of the electric field in array of optical waveguides with variable Kerr nonlinearity. In this context the role of time is played by the longitudinal propagation distance along the optical fiber and the nonlinear coefficients $\gamma_{i}, \gamma_{12}$ correspond to self- and cross-phase modulations of the electric field components, respectively [24, 25]. Notice that the above two component DNLSE has the Hamiltonian form $\dot{\chi}_{n}=\delta H / \delta \chi_{n}^{*}$ with $\chi_{n}=u_{n}, v_{n}$ and the Hamiltonian $H$ given by

$$
\begin{aligned}
H=- & \sum_{n}\left[\left(\kappa_{1} u_{n+1} u_{n}^{*}+\kappa_{2} v_{n+1} v_{n}^{*}+c . c .\right)+\right. \\
& \left.\frac{1}{2}\left(\gamma_{1}\left|u_{n}\right|^{4}+\gamma_{2}\left|v_{n}\right|^{4}\right)+\gamma_{12}\left|u_{n}\right|^{2}\left|v_{n}\right|^{2}\right] .
\end{aligned}
$$

Here the $*$ stands for the complex conjugation and c.c. denotes the complex conjugate of the expression in the parenthesis. Also notice that the number of atoms $N_{i}=\sum_{n}\left|\chi_{n}\right|^{2}, \quad \chi_{n}=u_{n}, v_{n}$ are conserved for each component.

Taking into account that Eq. (11) arises in the tight binding approximation [26, 27], the nonlinear coefficients $\gamma_{i}, \gamma_{12}$ can be expressed in terms of the overlap integrals of Wannier $W_{n}^{(i)}(x)$ functions of the corresponding continuous periodic Gross-Pitaevskii mean field model [28] as:

$$
\begin{aligned}
& \gamma_{12}=\frac{2 \pi \hbar \sqrt{N_{1} N_{2}} a_{12}}{m} \int d x\left|W_{n}^{(1)}\right|^{2}\left|W_{n}^{(2)}\right|^{2}, \\
& \gamma_{i}=\frac{4 \pi \hbar N_{i} a_{i i}}{m_{i}} \int d x\left|W_{n}^{i}\right|^{4}, \quad i=1,2,
\end{aligned}
$$

with $m_{i}, \quad i=1,2$ denoting the atomic masses and $m=m_{1} m_{2} /\left(m_{1}+m_{2}\right)$ the reduced mass (functions $W_{n}$ are normalized on the whole line: $\left.\int\left|W_{n}^{i}(x)\right|^{2} d x=1\right)$. The modulations of the nonlinear coefficients $\gamma_{i}, \gamma_{12}$ is assumed to be of the form

$$
\gamma_{i}(t)=\gamma_{i}^{(0)}+\frac{1}{\epsilon} \gamma_{i}^{(1)}\left(\frac{t}{\epsilon}\right)
$$

with $\gamma^{(0)}$ a constant, $\gamma^{(1)}(t)$ a rapidly varying periodic function of time and $\epsilon$ a small parameter that controls the strength and the frequency of the modulation (strong nonlinearity management corresponds to $\epsilon \ll 1$ ). For $\gamma_{i}^{(1)}(t)$ we consider periodic modulations of the type

$$
\gamma_{i}^{(1)}(t)=\frac{\gamma_{i}^{(1)}}{\epsilon} \cos \left(\Omega \frac{t}{\epsilon}\right), \quad \Omega, \gamma_{i}^{(1)} \sim O(1)
$$

of period $T=2 \pi / \Omega$ in the fast time variable $\tau=t / \epsilon$. Although in general one could consider independent modulations of the nonlinear coefficients, in practical contexts one usually deals with the simpler settings

$$
\begin{aligned}
& \text { - i) } \gamma_{i}=\gamma_{i}(t / \epsilon), \quad \gamma_{12}=\text { const }, \quad \mathrm{i}=1,2 \\
& \text { - ii) } \gamma_{12}=\gamma_{12}(t / \epsilon), \quad \gamma_{i}=\text { const }, \quad \mathrm{i}=1,2
\end{aligned}
$$

In the BEC context, case $i$ corresponds to a modulation in time of the intra-species scattering lengths, keeping inter-species scattering length constant, while in case $i i$ it is done just the opposite. Although these settings are both feasible for BEC mixtures, case ii) is not easy to implement in nonlinear optics because in this context cross-phase modulations are usually difficult to control. Moreover, the averaged equations obtained in the case ii) 
are mathematically more involved due to the presence of a complicated nonlinear dispersion (see below). In the following we concentrate mainly on case i) and discuss case ii) only briefly at the end of the section.

Let us consider then a fixed inter-species scattering length $\left(\gamma_{12}=\right.$ const $)$ and assume the intra-species scattering lengths modulated as follows:

$$
\gamma_{1}=\gamma_{1}^{(0)}+\gamma_{1}^{(1)}(t / \epsilon), \gamma_{2}=\gamma_{2}^{(0)}+\gamma_{2}^{(1)}(t / \epsilon)
$$

To find effective nonlinear evolution equations, we use averaging method to eliminate the fast time, $\tau=t / \epsilon$, dependence. In this respect it is convenient to perform the following transformation

$$
u_{n}=U_{n} e^{i \Gamma_{1}\left|U_{n}\right|^{2}}, v_{n}=V_{n} e^{i \Gamma_{2}\left|V_{n}\right|^{2}}
$$

where $\Gamma_{i}$ are the antiderivatives of $\gamma_{i}^{(1)}(t)$,

$$
\Gamma_{i}(t)=\int_{0}^{t} \gamma_{i}^{(1)}\left(t^{\prime}\right) d t^{\prime}-\frac{1}{T} \int_{0}^{T} \int_{0}^{t} \gamma_{i}^{(1)}\left(t^{\prime}\right) d t^{\prime} d t
$$

where $T$ is the period. By substituting Eq. (6) into Eqs.(1) we obtain:

$$
\begin{aligned}
i \dot{U}_{n}= & i \kappa_{1} \Gamma_{1}(\tau) U_{n}\left[U_{n}^{*} X_{1}-U_{n} X_{1}^{*}\right]- \\
& \kappa_{1} X_{1}-\left(\gamma_{1}^{(0)}\left|U_{n}\right|^{2}+\gamma_{12}\left|V_{n}\right|^{2}\right) U_{n} \\
i \dot{V}_{n}= & i \kappa_{2} \Gamma_{2}(\tau) V_{n}\left[V_{n}^{*} X_{2}-V_{n} X_{2}^{*}\right]- \\
& \kappa_{2} X_{2}-\left(\gamma_{2}^{(0)}\left|V_{n}\right|^{2}+\gamma_{12}\left|U_{n}\right|^{2}\right) V_{n}
\end{aligned}
$$

where $X_{1}=U_{n+1} e^{i \Gamma_{1} \theta_{1}^{+}}+U_{n-1} e^{i \Gamma_{1} \theta_{1}^{-}}, \quad X_{2}=$ $V_{n+1} e^{i \Gamma_{2} \theta_{2}^{+}}+V_{n-1} e^{i \Gamma_{2} \theta_{2}^{-}}$and

$$
\theta_{1}^{ \pm}=\left|U_{n \pm 1}\right|^{2}-\left|U_{n}\right|^{2}, \theta_{2}^{ \pm}=\left|V_{n \pm 1}\right|^{2}-\left|V_{n}\right|^{2} .
$$

The average over the rapid modulation can be done with the help of the relations

$$
\begin{aligned}
<e^{ \pm i \Gamma_{i} \theta^{ \pm}} & =J_{0}\left(\alpha_{i} \theta^{ \pm}\right) \\
<\Gamma_{i} e^{ \pm i \Gamma_{i} \theta^{ \pm}} & = \pm i \alpha_{i} J_{1}\left(\alpha_{i} \theta^{ \pm}\right)
\end{aligned}
$$

where $J_{0}, J_{1}$ are Bessel functions [29] of first kind of zeroth and first order, respectively, and with $\alpha_{i}$ given by

$$
\alpha_{i}=\gamma_{i} / \Omega, i=1,2
$$

Here the angular bracket denotes average with respect to the fast time variable, e.g. $\langle F\rangle \equiv(1 / T) \int_{0}^{T} F d \tau$. The system of averaged equations is then obtained as:

$$
\begin{aligned}
i \dot{U}_{n}= & -\alpha_{1} \kappa_{1} U_{n}\left[J_{1}\left(\alpha_{1} \theta_{1}^{+}\right)\left(U_{n}^{*} U_{n+1}+U_{n} U_{n+1}^{*}\right)\right. \\
& \left.+J_{1}\left(\alpha_{1} \theta_{1}^{-}\right)\left(U_{n}^{*} U_{n-1}+U_{n} U_{n-1}^{*}\right)\right] \\
& -\kappa_{1}\left[U_{n+1} J_{0}\left(\alpha_{1} \theta_{1}^{+}\right)+U_{n-1} J_{0}\left(\alpha_{1} \theta_{1}^{-}\right)\right] \\
& -\left[\gamma_{1}^{(0)}\left|U_{n}\right|^{2}+\gamma_{12}\left|V_{n}\right|^{2}\right] U_{n}, \\
i \dot{V}_{n}= & -\alpha_{2} \kappa_{2} V_{n}\left[J_{1}\left(\alpha_{2} \theta_{2}^{+}\right)\left(V_{n}^{*} V_{n+1}+V_{n} V_{n+1}^{*}\right)\right. \\
& \left.+J_{1}\left(\alpha_{2} \theta_{2}^{-}\right)\left(V_{n}^{*} V_{n-1}+V_{n} V_{n-1}^{*}\right)\right] \\
& -\kappa_{2}\left[V_{n+1} J_{0}\left(\alpha_{2} \theta_{2}^{+}\right)+V_{n-1} J_{0}\left(\alpha_{2} \theta_{2}^{-}\right)\right] \\
& -\left[\gamma_{2}^{(0)}\left|V_{n}\right|^{2}+\gamma_{12}\left|U_{n}\right|^{2}\right] V_{n} .
\end{aligned}
$$

Note that Eqs. (12 13) have Hamiltonian form with averaged Hamiltonian, $H_{a v}$, given by:

$H_{a v}=-\sum_{n}\left[\kappa_{1} J_{0}\left(\alpha_{1} \theta_{1}^{+}\right)\left[U_{n+1} U_{n}^{*}+c . c.\right]+\kappa_{2} J_{0}\left(\alpha_{2} \theta_{2}^{+}\right)\left[V_{n+1} V_{n}^{*}+c . c.\right]+\frac{1}{2}\left(\gamma_{1}^{(0)}\left|U_{n}\right|^{4}+\gamma_{2}^{(0)}\left|V_{n}\right|^{4}\right)+\gamma_{12}\left|U_{n}\right|^{2}\left|V_{n}\right|^{2}\right]$

By comparing Eq. (14) with the corresponding unperturbed Hamiltonian Eq. (2), one can see that the effect of the scattering lengths modulation simply reflects in the following nonlinear rescaling of the tunneling constants:

$$
\kappa_{i} \rightarrow \kappa_{i} J_{0}\left(\alpha_{i} \theta_{i}^{+}\right), i=1,2 .
$$

From this equation and from Eq. (11) it is clear that the inter-species interaction $\gamma_{12}$ play no role in determining the lattice sites where the zero tunneling condition occur (compacton boundaries) when the nonlinear management is made with respect to the intra-species interactions (see below for explicit examples). Thus, in analogy with the single component case considered in 1] the tunneling constants depend on the atom difference between neighboring sites [1, 2]. This introduces an effective nonlinear dispersion in the system which leads to the existence of two-component compactons.

In closing this section it is interesting to discuss the changes in the above derivation implied by a nonlinear management of the inter-species scattering length

$$
\gamma_{12}=\gamma_{12}^{(0)}+\gamma_{12}^{(1)}(t)
$$

with constant (not necessarily equal) intra-species parameters $\gamma_{1}, \gamma_{2}$. In this case, to remove the explicit time 
dependence from Eq. (1), the transformation in (6) must be replaced by

$$
u_{n}=U_{n} e^{i \Gamma(t)\left|V_{n}\right|^{2}}, v_{n}=V_{n} e^{i \Gamma(t)\left|U_{n}\right|^{2}},
$$

with $\Gamma(t)$ the antiderivative of $\gamma_{12}^{(1)}(t)$. Following the same approach as before, it is not difficult to show that one arrives at the same averaged Hamiltonian as in Eq. (14) but with the interchanges $\kappa_{1} \leftrightarrow \kappa_{2}, U \leftrightarrow V$, operated in the tunneling terms and the obvious replacements $\gamma_{12} \rightarrow$ $\gamma_{12}^{(0)}, \gamma_{i}^{(0)} \rightarrow \gamma_{i}$. This implies a different rescaling of the tunneling constants

$$
\kappa_{i} \rightarrow \kappa_{i} J_{0}\left(\alpha \theta_{3-i}^{+}\right), \quad i=1,2,
$$

and leads to complicated nonlinear dispersion terms in the averaged equations. In view of this complexity, in the following we restrict only to compactons induced by intra-species management. A detailed study of the interspecies management requires more investigations and will be discussed elsewhere [30].

\section{EXISTENCE AND STABILITY OF COMPACTONS IN BINARY BEC MIXTURES}

Exact compacton solutions of the averaged system can be searched as stationary states of the form:

$$
U_{n}=A_{n} e^{-i \mu_{u} t}, V_{n}=B_{n} e^{-i \mu_{v} t},
$$

with $\mu_{u}, \mu_{v}$ chemical potentials of the two atomic species. Substituting these expressions into Eq. (1213) one gets the following stationary equations:

$$
\begin{gathered}
\mu_{u} A_{n}+\left(\gamma_{1} A_{n}^{3}+\gamma_{12} B_{n}^{2} A_{n}\right)+\kappa_{1}\left[A_{n+1} J_{0}\left(\alpha_{1} \theta_{1}^{+}\right)+\right. \\
\left.A_{n-1} J_{0}\left(\alpha_{1} \theta_{1}^{-}\right)\right]+2 \alpha_{1} \kappa_{1} A_{n}^{2}\left[A_{n+1} J_{1}\left(\alpha_{1} \theta_{1}^{+}\right)+(20)\right. \\
\left.A_{n-1} J_{1}\left(\alpha_{1} \theta_{1}^{-}\right)\right]=0, \\
\mu_{v} B_{n}+\left(\gamma_{2} B_{n}^{3}+\gamma_{12} A_{n}^{2} B_{n}\right)+\kappa_{2}\left[B_{n+1} J_{0}\left(\alpha_{2} \theta_{2}^{+}\right)+\right. \\
\left.B_{n-1} J_{0}\left(\alpha_{2} \theta_{2}^{-}\right)\right]+2 \alpha_{2} \kappa_{2} B_{n}^{2}\left[B_{n+1} J_{1}\left(\alpha_{2} \theta_{2}^{+}\right)+(21)\right. \\
\left.B_{n-1} J_{1}\left(\alpha_{2} \theta_{2}^{-}\right)\right]=0,
\end{gathered}
$$

to be solved for the chemical potentials and amplitudes $A_{n}, B_{n}$ of the compacton modes. The compact nature of the solution $\left(A_{i}, B_{i}=0\right.$ outside a finite (small) range of sites), allows to truncate the above infinite system into a finite number of relations between the above variables, which can be solved exactly. In the following subsections this is shown explicitly for the different compacton types.

\section{A. Bright-Bright compactons}

To search compacton solutions of the B-B type we need to look for the last sites of vanishing amplitude, say $n_{0} \pm 1$,
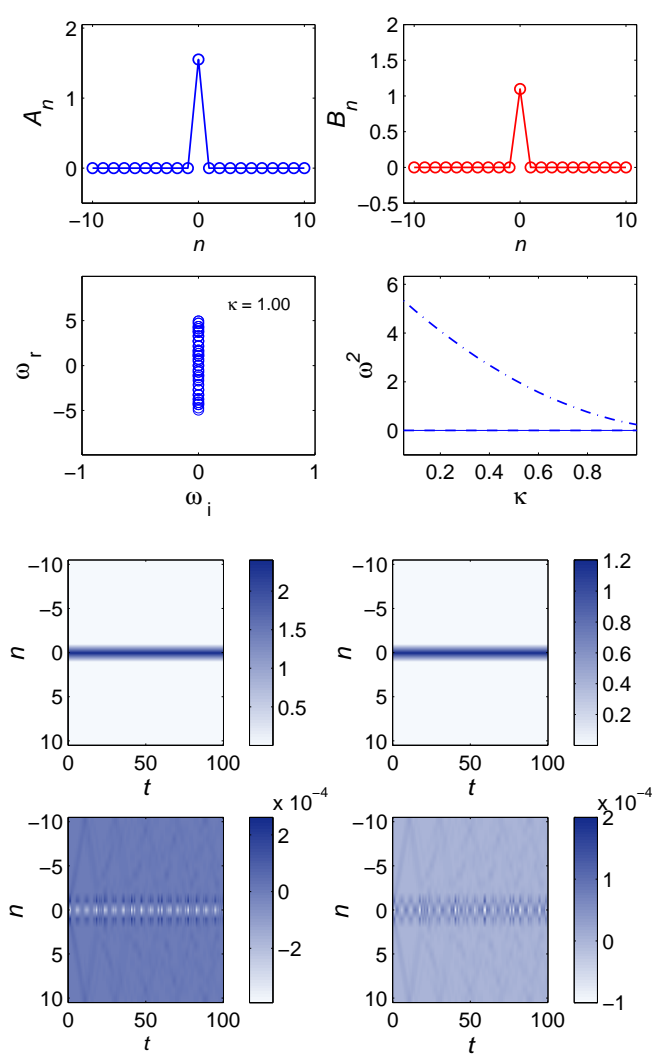

FIG. 1: (Color online) First row panels. Exact single site B-B compacton of Eqs. (12 13) for $\gamma_{1}=\gamma_{2}=1, \gamma_{12}=0.5$, $\kappa_{1}=\kappa_{2} \equiv \kappa=1, \alpha_{1}=1$, and $\alpha_{2}=2$. Second row panels. Real and imaginary parts of the eigenfrequency spectrum (left panel) corresponding to the B-B compacton depicted in top row panels. Right panel shows the lowest (solid line) third lowest (dash line) and fifth lowest (dash-dot line) $\omega^{2}$ values as a function of $\kappa$. Third row panels. Spacetime evolution of $\left|A_{n}\right|^{2}$ (left panel) and $\left|B_{n}\right|^{2}$ (right panel) as obtained from direct numerical integration of Eq. (1) with $\kappa_{i}=0.5, \gamma_{i}=1+\frac{\alpha_{i}}{\epsilon} \cos (t / \epsilon), i=1,2, \epsilon=0.01$, taking as initial condition the corresponding exact single site B-B compacton of the averaged system (other parameters are fixed as for top row panels). Bottom row panel. Deviation of the dynamics depicted in third row panels from the corresponding one obtained from Eqs. (12:13).

where the vanishing of the tunneling rate is realized. For a single site B-B compacton we assume $A_{n_{0}}=a, B_{n_{0}}=$ $b, A_{n_{0} \pm j}=0, B_{n_{0} \pm j}=0$ for all $j \geq 1$. Substituting this ansatz in Eqs.(2021) we obtain the corresponding condition for the compacton existence as

$$
\begin{gathered}
J_{0}\left(\alpha_{1} a^{2}\right)=0, a^{2}=\xi_{0} / \alpha_{1}, \\
J_{0}\left(\alpha_{2} b^{2}\right)=0, b^{2}=\xi_{0} / \alpha_{2}
\end{gathered}
$$

where $\xi_{0}$ is a zero of the Bessel function $J_{0}$ (in all numerical calculations below we take the first zero of $J_{0}$ : $\left.\xi_{0}=2.4048\right)$. This condition together with

$$
\mu_{u}=-\gamma_{1} a^{2}-\gamma_{12} b^{2}, \mu_{v}=-\gamma_{2} b^{2}-\gamma_{12} a^{2}
$$



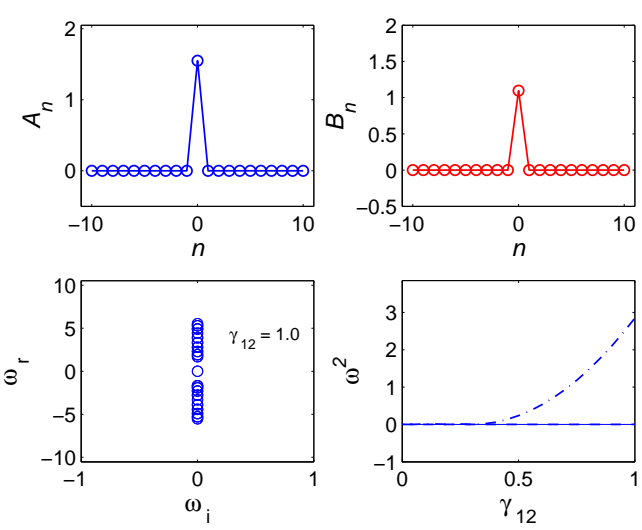

FIG. 2: (Color online) Same as first two rows panels of Fig. 1 except for compacton in top panels computed for $\gamma_{12}=1$ and stability properties in the bottom right panel computed as a function of $\gamma_{12}$. Other parameters are fixed as in Fig. 1.

gives us the single site $\mathrm{B}-\mathrm{B}$ compacton pair.

Typical examples of single site B-B compactons are depicted in top panels of Figs. 112 Stability properties of the solution have been investigated by standard linear analysis. Denoting by $\omega$ the eigenfrequencies of the linearized averaged equations, associated to growing perturbations of the form $e^{-i \omega t}$, we have that linear stability is granted if all $\omega$ have zero imaginary parts. For the considered single site B-B compacton, this condition is well satisfied, as one can see from the left panel in the second row of Fig. 1. Actually we find that single site B-B compactons are generically stable for wide range of parameters. This can be seen from the second top right panel of Fig. 1 1 where the dependence of the lowest squared eigenfrequencies, $\omega^{2}$, is reported as a function of $\kappa$ (to reduce number of parameters we fix, here and in the following, $\left.\kappa_{1}=\kappa_{2} \equiv \kappa\right)$. Extensive numerical linear stability studies show that $\omega^{2}$ is always non negative, meaning that the solution is linearly stable.

Nonlinear stability properties have been investigated by direct numerical integrations of Eqs. (12 13) taking as initial conditions exact compactons perturbed with a random uniformly distributed noise field of amplitude $10^{-4}$. Results are found in full agreement with the linear stability results discussed above. Excellent agreement is also obtained from direct numerical integrations of the original vector DNLSE (1), as one can see from third and fourth row panels of Fig. 1. In particular notice from the bottom row panels that the deviation of the original dynamics from the exact averaged dynamics is very small (four orders of magnitude small for $\epsilon=0.01$ ) and can be made even smaller by further decreasing $\epsilon$.

In Fig. 2 stability properties of single site B-B compactons have been investigated as a function of the interspecies interaction, $\gamma_{12}$, for a particular choice of the remaining parameters. As one can see, the obtained behavior is very similar to the one obtained for the $\kappa$ dependence in Fig. 1 this further confirming the robustness of
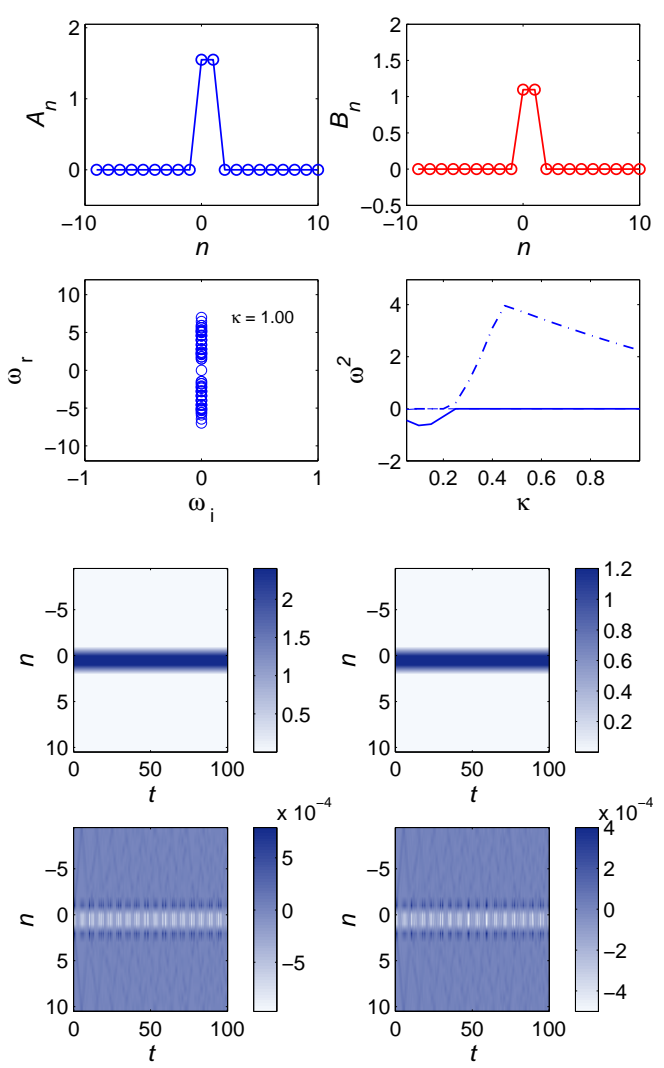

FIG. 3: (Color online) Same as in Fig. 1 but for a two-site in-phase B-B compacton. All parameters are fixed as in Fig. 1

the one site B-B solution with respect to wide parameters variations.

Two-site B-B compactons can also be found and, similarly to discrete breathers, can be of two types: in-phase and of out-of-phase. Two-site in-phase compactons follow from the ansatz: $A_{n_{0}}=a, A_{n_{0}-1}=0, A_{n_{0}+1}=$ $a, B_{n_{0}}=b, B_{n_{0}-1}=0, B_{n+1}=b$. By substituting into Eqs.(20121) one gets Eq. (22) as before with chemical potentials given by:

$$
\begin{aligned}
& \mu_{u}=-\gamma_{1} a^{2}-\gamma_{12} b^{2}-\kappa_{1}, \\
& \mu_{v}=-\gamma_{2} b^{2}-\gamma_{12} a^{2}-\kappa_{2} .
\end{aligned}
$$

First two row panels of Fig. 3 show a typical two-site inphase B-B stationary compacton. Notice from the second row panels that the linear eigenfrequencies are all positive in the interval $(0.25,1)$ this implying a wide stability rate also in this case. Direct numerical integrations of the averaged equation is shown in last two rows of Fig. 3.

Out-phase B-B compactons can be obtained from the ansatz $A_{n_{0}}=a, A_{n_{0}-1}=0, A_{n_{0}+1}=-a, B_{n_{0}}=$ $b, B_{n_{0}-1}=0, B_{n+1}=-b . \quad$ In this case the chemical potentials are given by

$$
\begin{aligned}
& \mu_{u}=-\gamma_{1} a^{2}-\gamma_{12} b^{2}+\kappa_{1}, \\
& \mu_{v}=-\gamma_{2} b^{2}-\gamma_{12} a^{2}+\kappa_{2},
\end{aligned}
$$



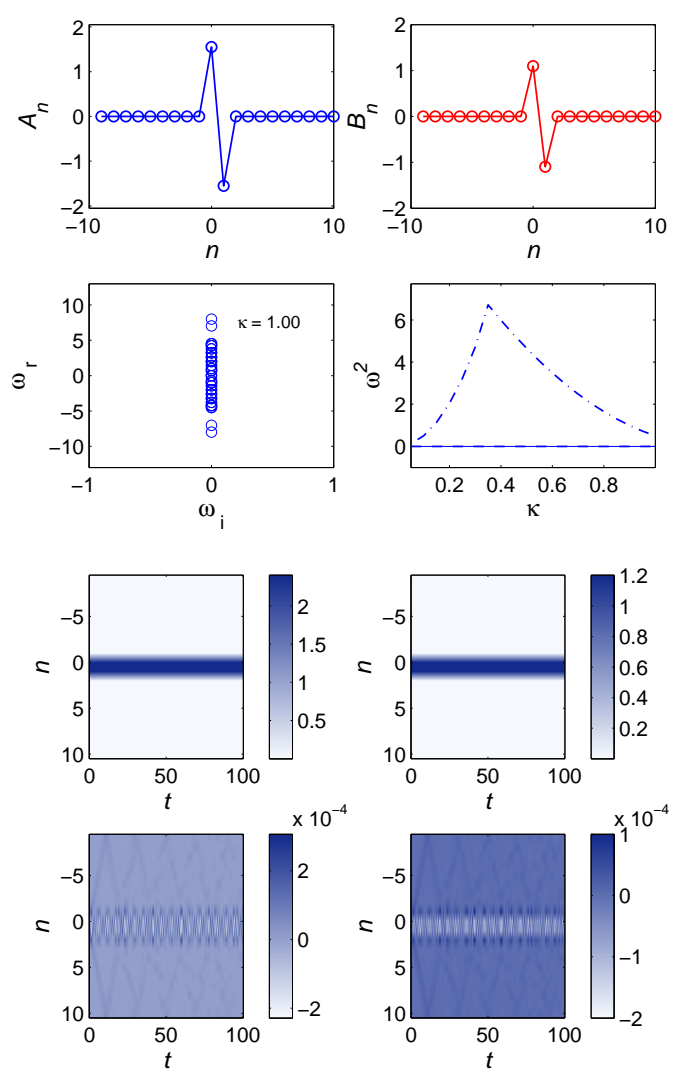

FIG. 4: (Color online) Same as in Fig. 1 but for a two-site out-of-phase B-B compacton. All parameters are fixed as in Fig. 1

with $a, b$, fixed as in Eq. (22). Typical two-site out-ofphase compacton and related linear stability analysis and time evolutions, are reported in Fig. 4.

Quite remarkably it is also possible to find exact threesite B-B compactons. In this case assuming $A_{n_{0}}=$ $a_{1}, A_{n_{0} \pm 1}=a_{2}, A_{n_{0} \pm 2}=0, B_{n_{0}}=b_{1}, B_{n_{0} \pm 1}=$ $b_{2}, B_{n \pm 2}=0$, we obtain chemical potentials as

$$
\begin{aligned}
\mu_{u}= & -\gamma_{1} a_{2}^{2}-\gamma_{12} b_{2}^{2}-\kappa_{1} a_{1} J_{0}\left(\xi_{u}\right) / a_{2} \\
& -2 \alpha_{1} \kappa_{1} a_{1} a_{2} J_{1}\left(\xi_{u}\right) \\
\mu_{v}= & -\gamma_{2} b_{2}^{2}-\gamma_{12} a_{2}^{2}-\kappa_{2} b_{1} J_{0}\left(\xi_{v}\right) / b_{2} \\
& -2 \alpha_{2} \kappa_{2} b_{1} b_{2} J_{1}\left(\xi_{v}\right)
\end{aligned}
$$

where $\xi_{u}=\alpha_{1}\left(a_{1}^{2}-a_{2}^{2}\right)$ and $\xi_{v}=\alpha_{1}\left(b_{1}^{2}-b_{2}^{2}\right)$.

The constraint equations for the amplitudes are Eq.(22) and

$$
\begin{array}{r}
\gamma_{1} a_{1} a_{2}\left(a_{2}^{2}-a_{1}^{2}\right)+\gamma_{12} a_{1} a_{2}\left(b_{2}^{2}-b_{1}^{2}\right) \\
+\kappa_{1}\left(2 a_{1}^{2}-a_{2}^{2}\right) J_{0}\left(\xi_{u}\right)+6 \alpha_{1} \kappa_{1} a_{1}^{2} a_{2}^{2} J_{1}\left(\xi_{u}\right)=0, \\
\gamma_{2} b_{1} b_{2}\left(b_{2}^{2}-b_{1}^{2}\right)+\gamma_{12} b_{1} b_{2}\left(a_{2}^{2}-a_{1}^{2}\right) \\
+\kappa_{2}\left(2 b_{1}^{2}-b_{2}^{2}\right) J_{0}\left(\xi_{v}\right)+6 \alpha_{2} \kappa_{2} b_{1}^{2} b_{2}^{2} J_{1}\left(\xi_{v}\right)=0 .
\end{array}
$$

Results are depicted in Fig. [5for typical example. Notice from second row right panel, that stability range in this
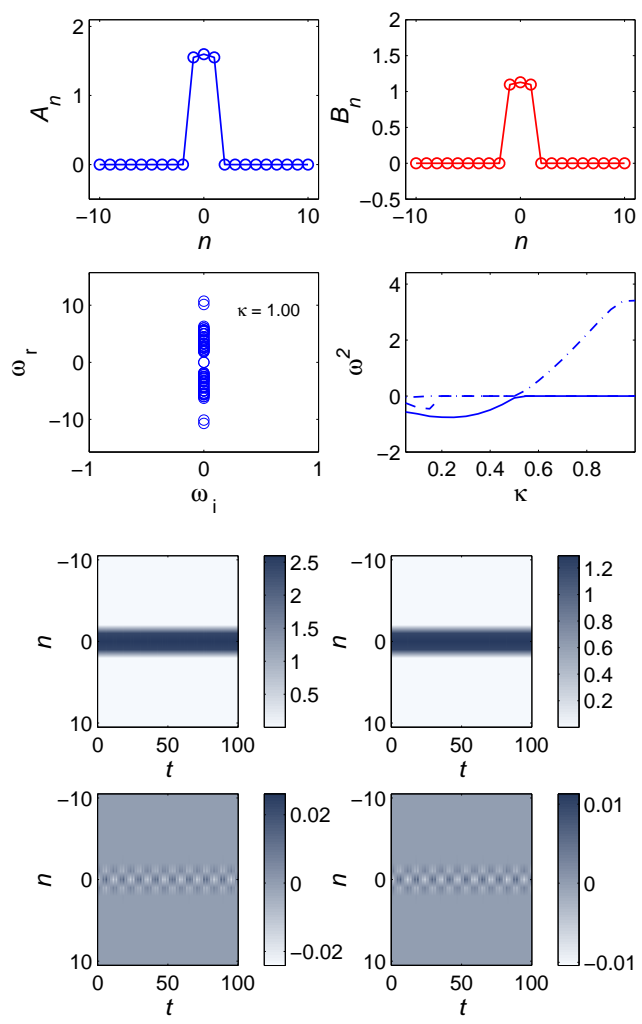

FIG. 5: (Color online) Same as in Fig. 1 but for a three-site B-B compacton. All parameters are fixed as in Fig. 1

case is strongly reduced and stability is possible only for $\kappa>\approx 0.5$. Also notice from the bottom panels that the deviation of the original dynamics relatively higher than the previous cases of B-B. The amplitude profile differs mainly at the middle point but this discrepancy, however, stays bounded in time and is quite small if compared to the maximum amplitudes of the exact solution for each component (for the considered case it never exceed the $2 \%$ of the exact amplitudes).

We also find that three-site B-B solutions with small $\alpha$ values display higher discrepancy compared to the respective solution with large $\alpha$ (by increasing $\alpha$, however, the amplitudes of the compacton also become smaller). These discrepancies may be ascribed to the management functions being not sufficiently strong. It is worth to remark here that in the limit $\epsilon \rightarrow 0$ (infinitely strong management) exact compactons of the averaged system should be exact also for Eq. (11) (limiting cases of very small $\epsilon$, however, are quite difficult to investigate numerically due to the very high accuracy required).

\section{B. Bright-Dark compactons}

It is interesting to look for novel type of solutions of the averaged DNLSE, which are more characteristic of the two-component systems, and in particular to B-D 

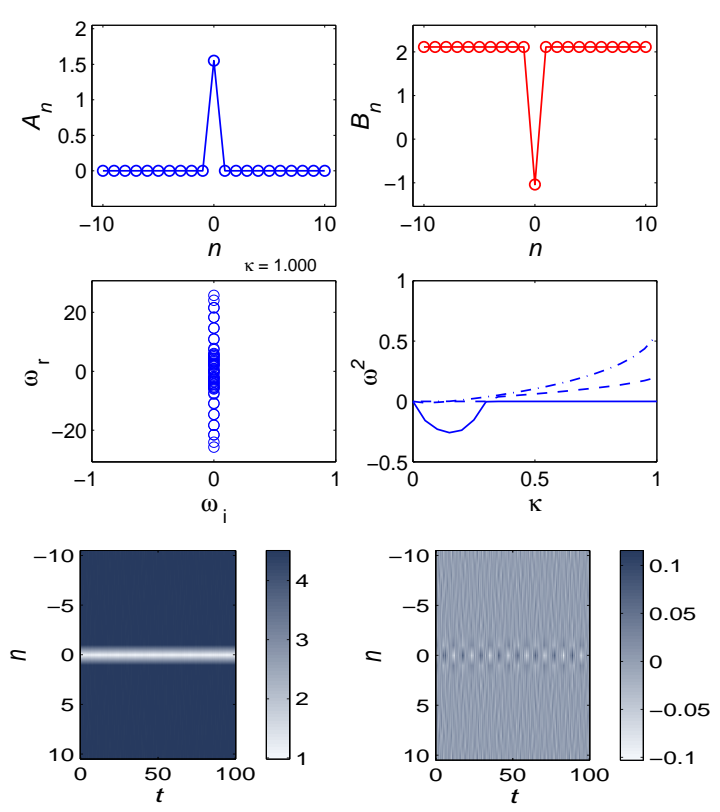

FIG. 6: (Color online) First row panels show the amplitude profile and second row left panel shows eigenfrequency spectrum of one site B-D compactons for case $\kappa=1, \gamma_{1}=$ $1, \gamma_{2}=-1, \gamma_{12}=-0.5, \alpha_{1}=1$, and $\alpha_{2}=1$. Second row right panel shows the numerical linear stability analysis as function of $\kappa$. Bottom panels show the space-time evolution (left panel) obtained from Eq. (1), of the dark component (square modulus) of the compacton depicted in top panels, and its deviation from the exact stationary solution (right panel). Modulation functions in Eq. (1) are taken as: $\gamma_{i}=1+\frac{\alpha_{i}}{\epsilon} \cos (t / \epsilon), \quad i=1,2$, with $\epsilon=0.01$.

(or dark-bright) onsite compactons. Existence of such solutions follows from the general stationary Eqs. 2021) by letting $A_{n_{0}}=a, B_{n_{0}}=b$, and $A_{n}=0, B_{n}=c$ for $n \neq n_{0}$. In this case one finds that exact solutions exist if chemical potentials and parameters $a, b, c$ of the B-D compacton are related by the following equations

$$
\begin{gathered}
a^{2}=\xi_{0} / \alpha_{1}, \quad \mu_{1}=-a^{2} \gamma_{1}^{0}-c^{2} \gamma_{12}, \quad \mu_{2}=-2 \kappa_{2}+b^{2} \gamma_{2}^{0}, \\
c J_{0}\left(\left(b^{2}-c^{2}\right) \alpha_{2}\right)-b\left(1+2 b c \alpha_{2} J_{1}\left(\left(b^{2}-c^{2}\right) \alpha_{2}\right)\right)=0, \\
2 b \kappa_{2} J_{0}\left(\left(b^{2}-c^{2}\right) \alpha_{2}\right)+c\left[\left(c^{2}-b^{2}\right) \gamma_{2}^{0}-2 \kappa_{2}+\right. \\
\left.\frac{\xi_{0}}{\alpha_{1}} \gamma_{12}+4 b c \kappa_{2} \alpha_{2} J_{1}\left(\left(b^{2}-c^{2}\right) \alpha_{2}\right)\right]=0,
\end{gathered}
$$

with $\xi_{0}$ a zero of $J_{0}$. The last equations can be solved numerically to determine the amplitude $b$ and the background $c$ of the dark component.

Typical one site B-D compactons are depicted in Figs. 617 (see top panels) together with their stability property (see left middle panels of these Fig.s). Stability properties versus $\kappa$ and $\gamma_{12}$ parameters are displayed in the second row right panels of Figs. 617 respectively, from which we see that in spite of the presence of some insta-
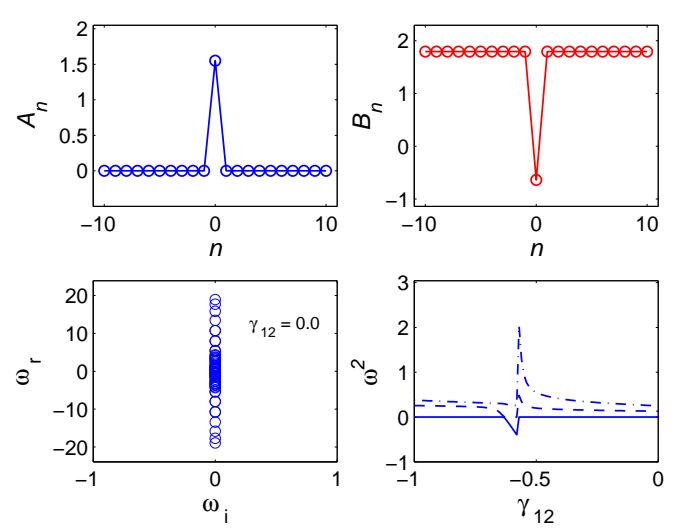

FIG. 7: (Color online) Same as first two rows panels of Fig. 6. except for one-site B-D compacton in top panels computed for $\gamma_{12}=0$ and stability properties in the bottom right panel computed as a function of $\gamma_{12}$. Other parameters are fixed as in Fig. 6]

bility region, the range of stability is still quite large. $\mathrm{Nu}-$ merical time evolution obtained from Eq. (11) and deviation from the exact stationary dynamics, are investigated for a B-D compacton taken in the left and right bottom panels of Fig. 6. The figure refers only to the dark component, being the bright component in very good agreement with the stationary solution. Notice from the right bottom panel the presence of fine periodical ripples on the background and small oscillations of the deep amplitude in the middle. These deviations, however, are relatively small compared to dark amplitude and background of the exact solution, remaining bounded on a long time scale.

\section{Dark-Dark Compactons}

Single site dark-dark (D-D) compactons can be searched of the form $A_{n_{0}}=b_{1}, B_{n_{0}}=b_{2}$ and $A_{n}=$ $a_{1}, B_{n}=a_{2}$ for $n \neq n_{0}$. The equations for chemical potential and amplitudes in this case are:

$$
\begin{gathered}
\mu_{u}=-\gamma_{1} a_{1}^{2}-\gamma_{12} a_{2}^{2}-2 \kappa_{1} \\
\mu_{v}=-\gamma_{2} a_{2}^{2}-\gamma_{12} a_{1}^{2}-2 \kappa_{2} \\
2 \kappa_{1} a_{1}\left(2 \alpha_{1} b_{1} J_{1}\left(\xi_{1}\right)+J_{0}\left(\xi_{1}\right) / b_{1}\right)-\gamma_{1}\left(a_{1}^{2}-b_{1}^{2}\right) \\
-\gamma_{12}\left(a_{2}^{2}-b_{2}^{2}\right)-2 \kappa_{1}=0 \\
2 \kappa_{2} a_{2}\left(2 \alpha_{2} b_{2} J_{1}\left(\xi_{2}\right)+J_{0}\left(\xi_{2}\right) / b_{2}\right)-\gamma_{2}\left(a_{2}^{2}-b_{2}^{2}\right) \\
-\gamma_{12}\left(a_{1}^{2}-b_{1}^{2}\right)-2 \kappa_{2}=0 \\
2 \alpha_{1} a_{1} b_{1} J_{1}\left(\xi_{1}\right)-b_{1} J_{0}\left(\xi_{1}\right) / a_{1}-1=0 \\
2 \alpha_{2} a_{2} b_{2} J_{1}\left(\xi_{2}\right)-b_{2} J_{0}\left(\xi_{2}\right) / a_{2}-1=0
\end{gathered}
$$

with $\xi_{i}=\alpha_{i}\left(a_{i}^{2}-b_{i}^{2}\right), i=1,2$.

The first row panels of Fig. 8 show the amplitude profile for $\gamma_{12}=0.2$ and respective numerical linear stability as function of $\kappa$. In the second row panels we report 

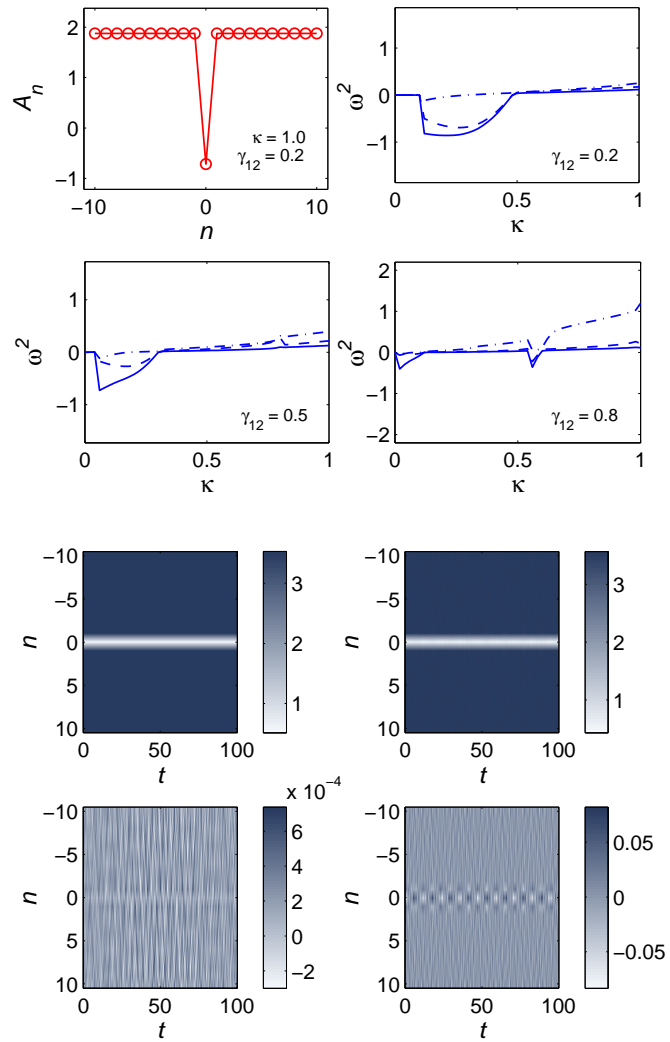

FIG. 8: (Color online) First row left panel shows the amplitude profile (identical for the two components) of a single site D-D compacton for parameter values $\kappa=1, \gamma_{1}=\gamma_{2}=-1$, $\gamma_{12}=0.2, \alpha_{1}=1$, and $\alpha_{2}=1$. In the right panel the numerical linear stability is reported as as function of $\kappa$. Second row panels show linear stability analysis for cases $\gamma_{12}=0.5$ (left) and $\gamma_{12}=0.8$ (right). Third row panels show the space-time dynamics (only one component shown) as obtained from Eqs. (1213) (left) and Eq. (1) (right), for a D-D compacton with parameters fixed as in top panel but with $\gamma_{12}=0.5$. Bottom panels show the deviation of the corresponding above dynamics from the exact solution.

stability properties for two increasing values of the interspecies interaction: $\gamma_{12}=0.5,0.8$ (left and right panel, respectively). As one can see, the $\kappa$ region of stability becomes wider as $\gamma_{12}$ is increased, this indicating a tendency of the coupling to stabilize dark-dark pairs. Time evolutions with respect to the averaged and original systems and their deviations from the exact solution are shown in the bottom two rows left and right panels, respectively. In general we find that D-D system is more sensitive to disturbance compared to all previous cases and for higher value of $\kappa$ and $\gamma_{12}$ (such as $\kappa=1$ and $\gamma_{12}=0.8$, for example) much smaller $\epsilon$ and numerical time step are needed to numerically simulate stable dynamics on a long time scale (exact initial solutions can be perturbed only by very small noise of the order $10^{-6}$ ). A possible reason for this weak stability is discussed in the next section.
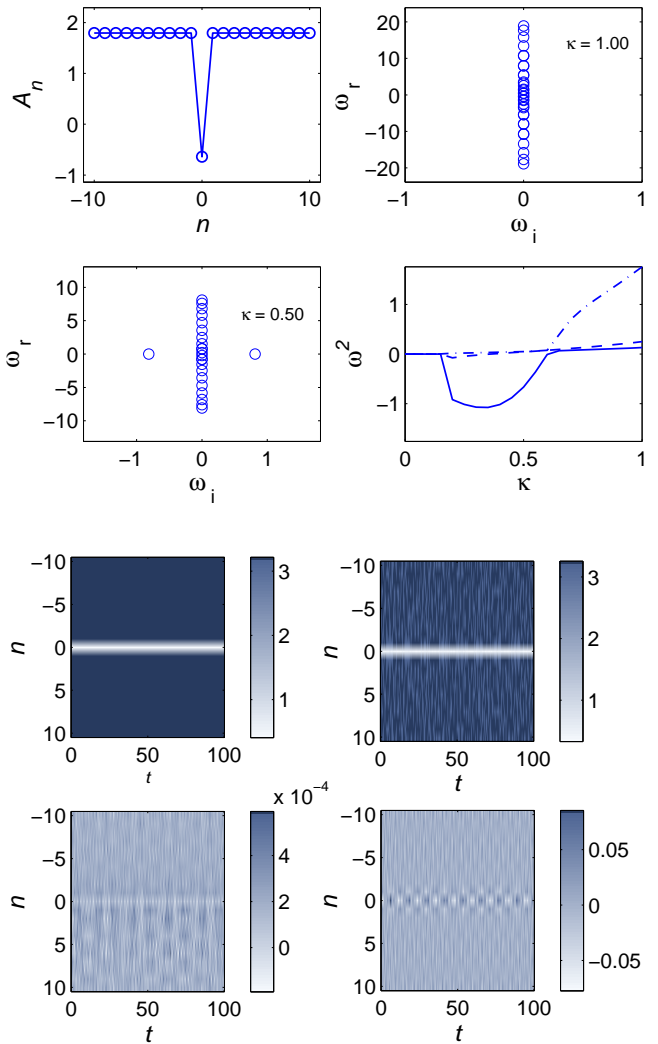

FIG. 9: (Color online) First row panels show the amplitude profile (left) and eigenfrequency spectrum (right) of uncoupled one site dark compacton for case $\kappa=1, \gamma_{0}=1$ and $\alpha=1$. Second row panels show eigenfrequency spectrum for case $\kappa=0.5$ (left) and the numerical linear stability analysis as function of $\kappa$ (right). Last two row panels. Space-time evolution of one site dark compacton solution for parameter case as in upper panels. Third row panels show the square modulus of the solution, the left side from the averaged system Eq.1213) and the right side from Eq.(1), while last row panels show their respective deviations from the exact solution.

\section{SINGLE COMPONENT DARK COMPACTON}

From the above analysis it appears evident that stability properties become more critical for compactons that involve dark components. This is true for B-D and even more for D-D pairs. This fact may result from a possible instability of the single component dark compacton. We remark that for single component BEC, compactons were investigated only for the bright case [1], so the question of whether stable single component dark compactons can exist, is open. In this section we provide the answer to the question by studying the uncoupling limit $\gamma_{12} \rightarrow 0$ of the D-D compacton discussed before.

In this respect the averaged equations in (12/13) for $\gamma_{12}=0$ with $\kappa_{i} \equiv \kappa, \alpha_{i} \equiv \alpha, \theta_{i, \pm} \equiv \theta_{ \pm}$reduce to the same equations considered in [1] for the single field variable 
$U_{n}=V_{n} \equiv u_{n}$. Considering $u_{n}=A_{n} \exp (-i \mu t)$ then the equation becomes

$$
\begin{aligned}
\mu A_{n} & =-2 \kappa \alpha A_{n}^{2}\left[A_{n+1} J_{1}\left(\alpha \theta^{+}\right)+A_{n-1} J_{1}\left(\alpha \theta^{-}\right)\right] \\
& -\kappa\left[A_{n+1} J_{0}\left(\alpha \theta^{+}\right)+A_{n-1} J_{0}\left(\alpha \theta^{-}\right)\right]+\gamma_{0} A_{n}^{3}
\end{aligned}
$$

with $\theta^{ \pm}=A_{n \pm 1}^{2}-A_{n}^{2}$. Dark modes are possible only for repulsive interactions so that in the above equation $-\gamma_{1}^{(0)} \equiv \gamma_{0}>0$. A dark single site compacton located at the $n=n_{0}$ site can be determined by setting $A_{n}=b$ if $n=n_{0}$ and $A=a$ for $n \neq n_{0}$ to yield

$$
\begin{aligned}
& 2 a \kappa J_{0}(\xi)+b\left[\left(a^{2}-b^{2}\right) \gamma_{0}-2 \kappa+4 a b \kappa \alpha J_{1}(\xi)\right]=0 \\
& b J_{0}(\xi)-a\left(1+2 a b \alpha J_{1}(\xi)\right)=0
\end{aligned}
$$

where $\xi=\alpha\left(a^{2}-b^{2}\right)$. It follows that to satisfy the equation at the nonvanishing sites, one must have

$$
\mu=a^{2} \gamma_{0}-2 \kappa .
$$

then Eq.(28) can be numerically solved to obtain $\{a, b\}$.

Numerical linear stability analysis for parameters values $\gamma_{0}=1$ and $\alpha=1$ (see second row, right panel of Fig. 9) shows that the solutions are unstable until $\kappa>0.65$. A comparisons with the first two rows of Fig. 8 indicates that the stability regime can be improved by inter-species coupling in the two component DNLS system. Nevertheless, when $\kappa>0.65$, stable dark compactons exist as shown in the third row of Fig. 9 for case $\kappa=1$.

From this it appears evident that, in contrast with the bright compactons case, the strong nonlinear management does not provide (at least for the simple real amplitudes ansatz assumed) stable single component dark compacton solutions for wide range of parameters. It is interesting that this situation is slightly improved when the inter-species interaction is switched on, as discussed before.

\section{GENERATION OF BINARY BEC COMPACTONS AND EXPERIMENTAL SETTING}

It is also interesting to discuss physical conditions for which compactons could be experimentally observed in ultracold BEC mixtures. From a first sight one could think that, in order to keep the zero tunneling condition satisfied at the edges one needs a very precise control of the number of atoms inside the compacton, a fact that could be difficult to arrange in a real experiments. On the other hand, if the excitation is very stable, it should appear also in the presence of generic fluctuations that are unavoidable in any real experiment. In this respect is of interest to address the problem of compacton generation from generic initial excitations, this providing evidence of their robust emergence even under unfavorable conditions. To this end, let us concentrate on the most stable compact excitations, which, as we have seen, are
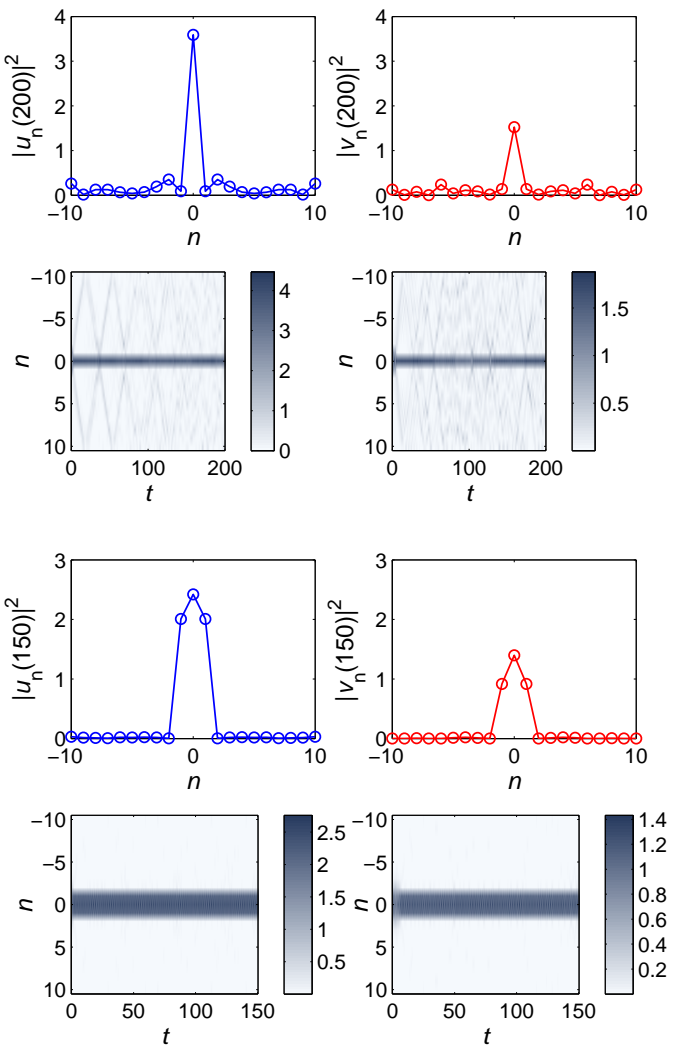

FIG. 10: (Color online) Top two row panels. Single site B$\mathrm{B}$ compacton emerging from the time evolution of an initial Gaussian excitation (described in the text) of Eq. (1) with nonlinear management function taken as in Fig. 6 but with $\epsilon=0.1$. Parameter are fixed as $\kappa=0.5, \alpha_{1}=1, \alpha_{2}=2, \gamma_{1}=$ $\gamma_{2}=1, \gamma_{12}=0.5$. Top panels show the density profiles of the first (left) and second (right) component at time $t=200$ while second row panels show the corresponding time evolutions. Bottom two row panels. Same as in top two row panels but for $\epsilon=0.01$, and $\kappa=1$ and for a three site B-B compacton displayed at time $t=150$. Other parameters are as in top two row panels.

the ones of B-B type. Without loss of generality, we take initial excitations of Gaussian-type for both components $u_{n}(0)=A \exp \left(-\eta n^{2}\right) / \alpha_{1}, \quad v_{n}(0)=A \exp \left(-\eta n^{2}\right) / \alpha_{2}$, with $A, \eta$ fixed as $A=1.55, \eta=0.2$ in the following numerical simulations (similar results can be obtained with other types of initial conditions).

In Fig. 10 we show the generation of a single site (see top two row panels) and a three sites (see bottom two row panels) B-B compacton from a Gaussian initial condition (same for both cases). The first and third row panels refer the density profiles (at time $t=200$ and $t=150$, respectively) while second and forth row panels refer to time evolutions of the two components. From top two row panels we see that after expelling some excess matter, a single site compacton on site $n=0$ (plus some background noise), emerge. The background noise is unavoidable because the number of atoms (squared 

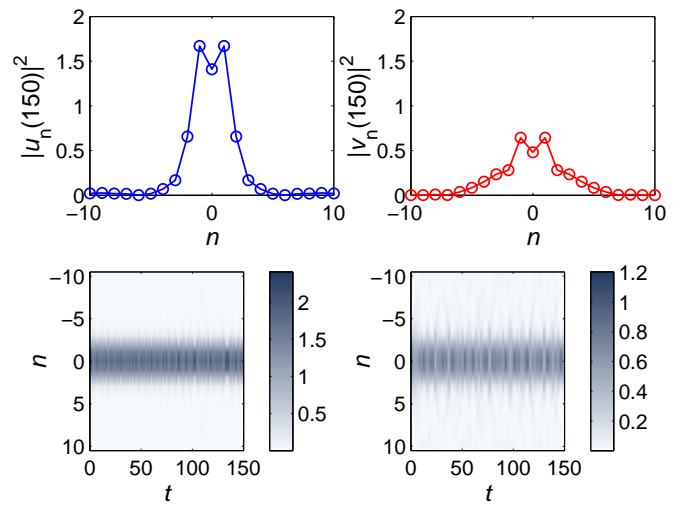

FIG. 11: (Color online) Same as in bottom two row panels of Fig. 10 but for $\gamma_{12}=0.2$.

amplitudes) in the compacton for the chosen parameter is fixed by the first zero of $J_{0}$ and is smaller than the number of atoms in the initial Gaussian. In spite of this, the densities of the two components in the neighboring sites, $n= \pm 1$, keep very small (although not exactly zero) during their time evolution, irrespectively from the excess radiation. For the considered case, $\epsilon$ was 0.1 and therefore the management was not very strong. One can expect that by further decreasing $\epsilon$ one can better and better approximate a true compacton solution with vanishing densities at $n= \pm 1$ (this becoming exactly true in the limit $\varepsilon \rightarrow 0$ ). The effect of a stronger management can be see from the bottom two row panels of Fig. 10. In this case epsilon is one order of magnitude smaller $(\epsilon=0.01), \kappa=1$ and the mismatch between numbers of atoms in the initial and final states is quite small. As a result, we see that a three site compacton, practically indistinguishable from an exact solution based on the first zero of $J_{0}$, is formed. This clearly demonstrates that, with proper parameter design and proper management conditions, compactons can be very robust excitations that can emerge spontaneously from generic initial conditions.

Further results shown in Fig. 11 address the problem of the influence of the inter-species interaction $\gamma_{12}$ on the compacton formation. Here same parameters and same Gaussian initial excitations as in bottom two row panels in Fig. 10 are used, but with lower value of the interspecies interaction, $\gamma_{12}=0.2$. In this case we see that instead of a three site compacton, an intrinsic localized mode with exponential tail is formed, in full agreement with the fact that by lowering the inter-species interaction the compacton stability may be lost.

Let us now discuss possible experimental setting for the observation of compacton modes. In this respect we remark that the existence bound pairs of B-D solitons (localized excitation with tails) has been experimentally demonstrated in 23] for the continuous case. A possible experimental setting to observe the corresponding compacton modes could be a BEC mixture of ${ }^{41} \mathrm{~K}$ and ${ }^{87} \mathrm{Rb}$ atoms loaded in a deep optical lattice considered in the experiment [31] and subjected to inter-species scattering length modulations. An equivalent setting could be implemented also in nonlinear optics with an array of optical wave-guides with varying Kerr nonlinearity along the propagation [24, 32]. For the case of binary BEC mixtures, the time modulations of the scattering lengths can be easily implemented by means of the FR technique by varying the external magnetic field, $B$, near a resonant value:

$$
a_{i j}(t)=a_{i j, b r}\left(1-\frac{\Delta}{B_{i j, 0}-B(t)}\right) .
$$

Here $a_{i j, b r}$ denotes the background value of the respective scattering lengths and $B_{i j, 0}$ the resonant value of the magnetic field. In the case of ${ }^{87} \mathrm{Rb}$ atoms, for example, there are several narrow FR which could be used, the broadest one lying at $B_{0}=1007 \mathrm{G}$. The tight-binding limit, appropriate for a vector DNLSE description, could be reached by considering optical lattices of amplitude $V_{0}>10 E_{R}$, where $E_{R}=\hbar^{2} k^{2} / 2 m$ is the recoil energy. Thus, by changing periodically and rapidly in time the magnetic field around a FR of the intra-species scattering lengths it should be possible to observe two-component matter wave compactons to emerge from generic initial conditions in real experiments.

\section{CONCLUSIONS}

In conclusion, we have investigated the existence and stability of binary mixtures matter waves in arrays of BEC subjected to time dependent periodic variations of the scattering length, by means of an averaged twocomponent DNLSE. In addition of B-B compactons we showed that B-D and D-D compactons are also possible. The stability of these modes has been investigated both by linear spectral analysis and by direct numerical integrations. We found that the single site and the two-site (out-of-phase) B-B compactons are always very stable in the whole parameter range, while for the other modes there exist thresholds in the tunneling constant rate below which they cannot exist as stable excitations. The stability resulted in general to be more critical for pairs involving one (or both) component of dark type. In all cases, however, the predictions of the averaged system were found in good agreement (in some cases; excellent) with the results of the numerical simulations.

\section{Acknowledgements}

M. S. acknowledges partial support from the Ministero dell'Istruzione, dell'Universitá e della Ricerca (MIUR) through a PRIN (Programmi di Ricerca Scientifica di Rilevante Interesse Nazionale) 2010-2011 initiative. 
[1] F. Kh. Abdullaev, P. G. Kevrekidis, and M. Salerno, Phys.Rev.Lett. 105, 113901 (2010).

[2] J. Gong, L. Morales-Molina, and P. Hänggi, Phys. Rev. Lett. 103, (133002) (2009).

[3] S. Inouye, M. R. Andrews, J. Stenger, H.-J. Miesner, D. M. Stamper-Kurn and W. Ketterle, Nature 392, 151 (1998).

[4] S. Greschner, G. Sun, D. Poletti, and L. Santos, Phys. Rev. Lett. 113, 215303 (2014).

[5] A. Rapp, X, Deng, and L. Santos, Phys.Rev.Lett. 109, 203005 (2012).

[6] T. Wang, X.-F. Zhang, F. E. A. dos Santos, S. Eggert, and A. Pelster, Phys. Rev. A 90013633 (2014).

[7] M. Di Liberto, C. E. Creffield, G. I. Japaridze, and C. M. Smith, Phys. Rev. A 89, 013624 (2014).

[8] S. Greschner, L. Santos, and D. Poletti, Phys. Rev. Lett. 113, 183002 (2014).

[9] H. Saito, M. Ueda, Phys. Rev. Lett. 90, 040403 (2003).

[10] F. Kh. Abdullaev, J.G. Caputo, R.A. Kraenkel, and B.A. Malomed, Phys. Rev. A 67, 013605 (2003).

[11] G. D. Montesinos, V. M. Perez-García and P. Torres, Physica D 191, 193 (2004).

[12] P. Kevrekidis, D. Pelinovsky, A. Stefanov, J. Phys. A 39, 479 (2006).

[13] F. Kh. Abdullaev, J. Garnier, Phys. Rev. E 72, 035603(R) (2005).

[14] M. Salerno, V.V. Konotop and Y.V. Bludov, Phys. Rev. Lett. 101, 030405 (2008).

[15] C. Gaul, R.P.A. Lima, E. Díaz, C.A. Müller, and F. Dominguez-Adame, Phys.Rev.Lett. 102, 255303 (2009).

[16] E. Díaz, C. Gaul, R. P. A. Lima, F. Dominguez-Adame, and C. A. Müller, Phys.Rev. A 81, 051607 (2010).

[17] Yu. Bludov, V.V. Konotop and M. Salerno, Europhys. Lett. 87 (2009) 20004.
[18] Yu. Bludov, V.V. Konotop and M. Salerno, Phys.Rev.A. 80 023623, (2009)

[19] K. Staliunas, S. Longhi, and G. J. de Valcarcel, Phys. Rev. Lett. 89, 210406 (2003).

[20] F.Kh. Abdullaev, M. Ogren, and M.P. Soerensen, Phys. Rev. A 87, 023616 (2013).

[21] I. Gabitov and S. K. Turytsin, Opt. Lett. 21, 327 (1996); N. J. Smith et al., Electron. Lett. 32, 54 (1996).

[22] Yu. Bludov, V.V. Konotop and M. Salerno, Europhys.Lett. 93, 30003 (2011).

[23] U. Shrestha, J. Javanainen, and J. Ruostekoski, Phys.Rev.Lett. 103, 190401 (2009).

[24] Gaetano Assanto, Luis A. Cisneros, Antonmaria A. Minzoni, Benjamin D. Skuse, Noel F. Smyth, and Annette L. Worthy, Phys.Rev.Lett. 104, 053903 (2010).

[25] A. Kobyakov, S. Darmanyan, F. Lederer, E. Schmidt, Optical and Quantum Electronics 30, 795 (1998).

[26] A. Trombettoni and A. Smerzi, Phys. Rev. Lett. 86, 2353 (2001).

[27] F.Kh. Abdullaev, B. B. Baizakov, S. A. Darmanyan, V. V. Konotop, and M. Salerno, Phys. Rev. A 64, 043606 (2001).

[28] G.L. Alfimov, P.G. Kevrekidis, V.V. Konotop, and M. Salerno, Phys. Rev E 66046608 (2002).

[29] M. Abramowitz and I. A. Stegun, Handbook of Mathematical Functions, (National Bureau of Standards, Washington, 1964).

[30] F.Kh. Abdullaev, M.S.A. Hadi, M. Salerno, and B. Umarov, unpublished.

[31] J. Catani, L. De Sarlo, G. Barontini, F. Minardi, and M. Inguscio, Phys. Rev. A 77, 011603(R) (2008).

[32] F. Kh. Abdullaev, E. N. Tsoy, B. A. Malomed, and R. A. Kraenkel, Phys. Rev. A 68, 053606 (2003). 